
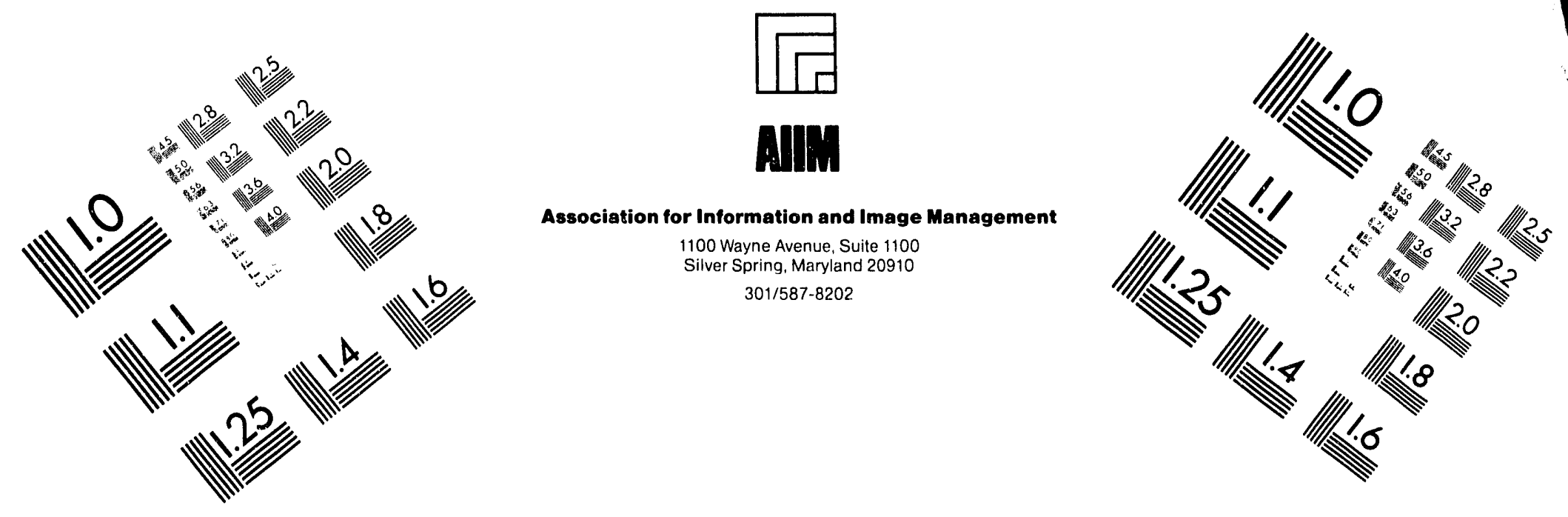

Centimeter

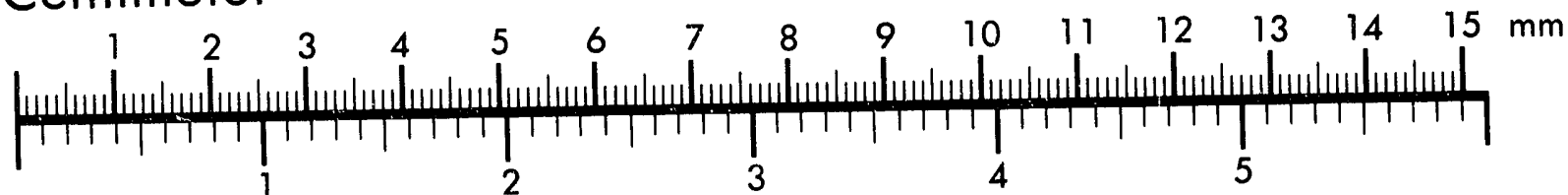
Inches
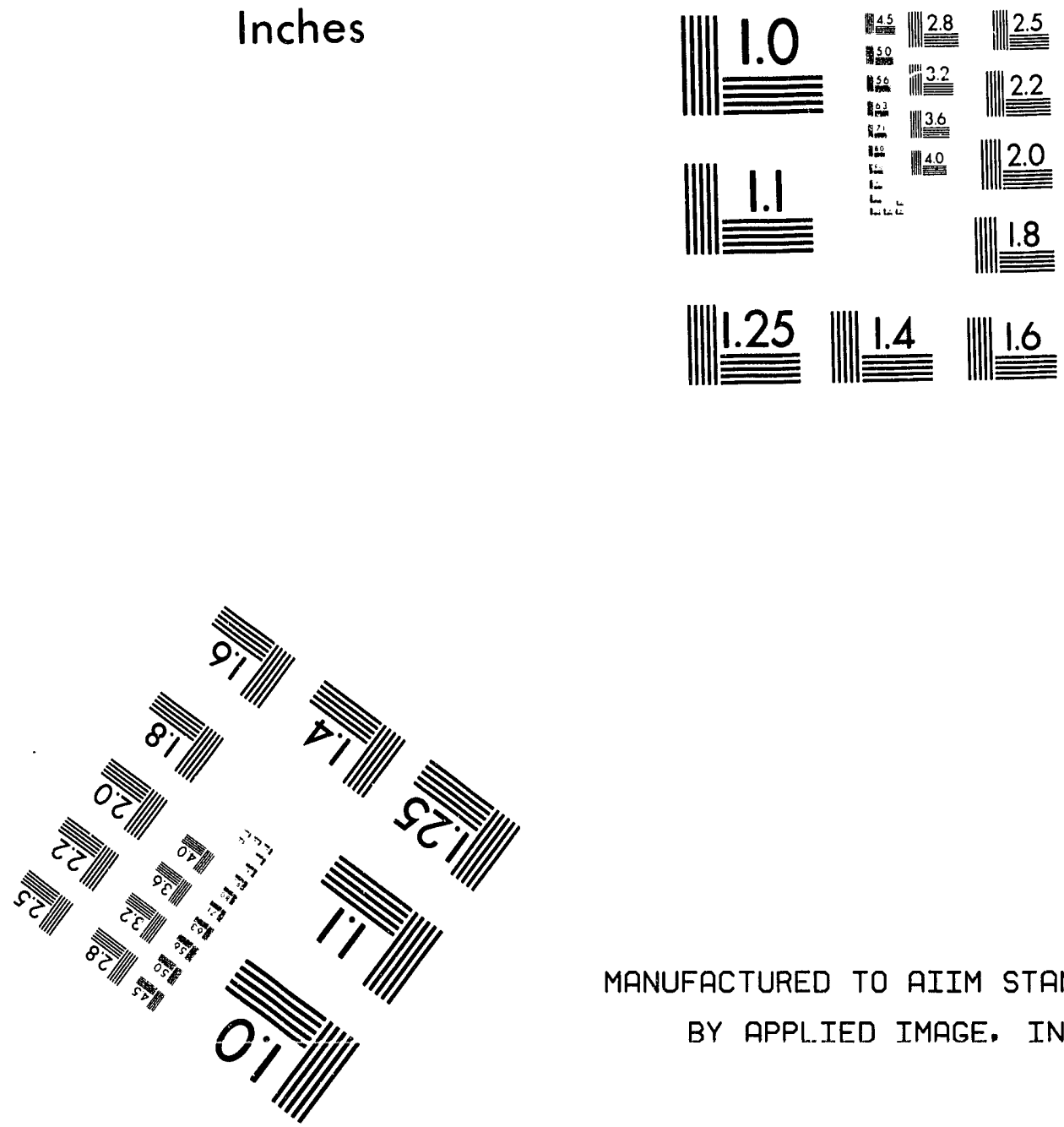

MANUFACTURED TO AIIM STANDARDS

BY APPL.IED IMAGE. INC.

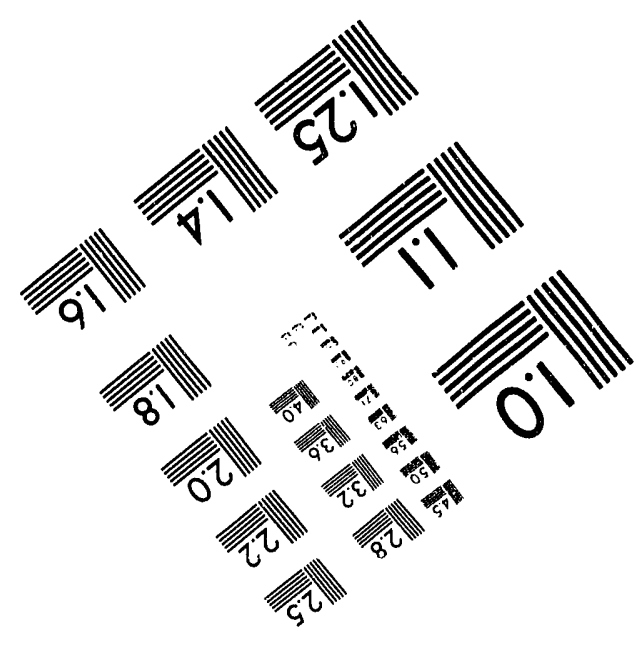



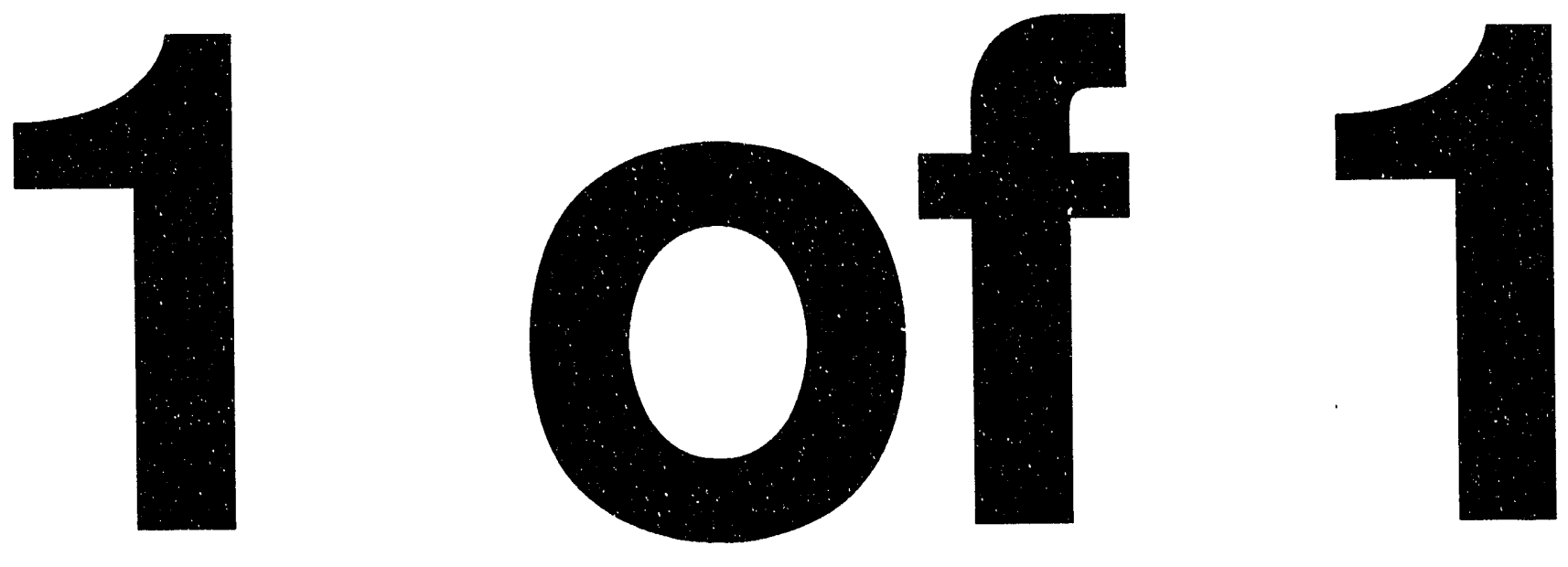


\title{
A Multiphase Model for Shock-Induced Flow in Low Density Foam
}

\author{
M. R. Baer, \\ Energetic Materials and Fluid Mechanics Dept. 1512 \\ Sandia National Laboratories, Albuquerque,NM
}

\begin{abstract}
A multiphase mixture model is applied to describe shocked-induced flow in deformable low-density foam. This model incle'des interphase drag and heat transfer and all phases are treated as compressible. Volume fraction is represented as an independent kinematic variable and the entropy inequàlity suggests a thermodynamically-admissable evolutionary equation to describe rate-dependent compaction. This multiphase model has been applied to shock tube experiments conducted by B. W. Skews and colleagues in the study of normal shock impingement on a wall-supported low density porous layer. Numerical solution of the multiphase flow equations employs a high resolution adaptive finite element method which accurately resolves contact surfaces and shock interactions. Additional studies are presented in an investigation of the effect of initial gas pressure in the foam layer, the shock interaction on multiple layers of foam and the shock-induced flow in an unsupported foam layer.
\end{abstract}

\section{Introduction}

In contrast to single-phase materials, porous materials contain internal boundaries which greatly influence the thermal and mechanical response to shock loading. In spite of the wealth of study in multiphase flow, much remains to be understood regarding the complex nature of shock-induced flow in distended materials. Continuum mechanics has provided a foundation for theoretical study and mixture theory has successfully described various multiphase waves ${ }^{1,2}$; however, much of this analysis has focussed on the strong shock wave response of high density porous materials. This study extends the application of mixture theory to shock-induced flow in low density foam.

In recent years, B.W. Skews and colleagues ${ }^{3,4}$ have reported shock tube experiments of wallsupported low density foams. Pressure measurements verified that dispersive waves are produced in the foam material following shock impingement and stagnation of material causes pressure amplification at the wall. As the foam material reflects off the wall, additional waves are observed. This study provides a theoretical description which replicates the observations of these experiments and complements a study of shock interactions on multiple foam layers. Additionally, normal shock impingement on an unsupported porous layer has been modeled and compared to the experimental work of Onodera, et al ${ }^{5}$.

\section{Theoretical Description}

The continuum mixture model used in this study is taken, in its entirety, from the work of Baer and Nunziato ${ }^{1}$; only the final forms of this model are given here. For simplicity, the balance equations are given for one-dimensional two-phase flow. Thus, the effects of material strength and wall boundary friction are not included. Each phase is denoted with a subscript " $a$ " to identify solid $(a=s)$ and gas phase $(a=g)$. Associated with each phase are the material densities, $\rho_{a}$, volume fractions, $\phi_{a}$, particle velocities, $v_{a}$, pressures, $p_{a}$, temperatures, $T_{a}$, and internal energies, $e_{a}$. The Eulerian form of the balance laws, excluding the effects of mass exchange, are given as:

Mass:

$$
\frac{\partial}{\partial t}\left(\phi_{a} \rho_{a}\right)+\frac{\partial}{\partial x}\left(\phi_{a} \rho_{a} v_{a}\right)=0
$$

Momentum:

$$
\begin{gathered}
\frac{\partial}{\partial t}\left(\phi_{a} \rho_{a} v_{a}\right)+\frac{\partial}{\partial x}\left(\phi_{a} p_{a}+\phi_{a} \rho_{a} v_{a}^{2}\right)=m_{a}^{\ddagger} \\
\frac{\partial}{\partial t}\left(\phi_{a} \rho_{a} E_{a}\right)+\frac{\partial}{\partial x}\left(\left(\phi_{a} \rho_{a} E_{a}+\phi_{a} p_{a}\right) v_{a}\right)=e_{a}^{\ddagger}
\end{gathered}
$$

where $E_{a}=e_{a}+v_{a}^{2} / 2$ and the " $\ddagger$ " superscript denotes a phase exchange quantity.

To describe the rate of change of solid volume fraction, a compaction model is derived from the entropy inequality given as:

$$
\frac{\partial \phi_{s}}{\partial t}+v_{s} \frac{\partial \phi_{s}}{\partial x}=\frac{\phi_{s} \phi_{g}}{\mu_{c}}\left(p_{s}-p_{g}-\beta_{s}\right)
$$

and $\phi_{s}+\phi_{g}=1$ is the saturation constraint. The intragranular stress, $\beta_{s}$, is the stress associated with the contact forces of the solid phase pore structure and is a different stress state than the bulk stress associated with the material compressibility. The compaction viscosity, $\mu_{c}$, dictates the rate at which

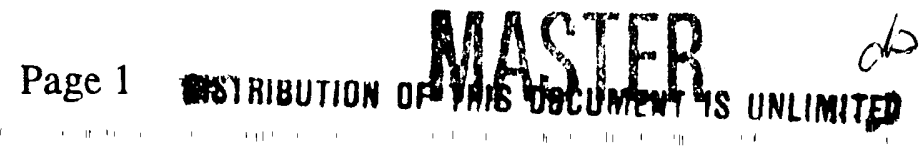


the volume fraction adjusts to an equilibrium stress state: $p_{s}=p_{8}+\beta_{s}$, and its value is estimated to be $O\left(10^{2}-10^{4}\right)$ [poise] as determined in prior dynamic compaction studies ${ }^{6}$.

Consistent with the constraints of the Second Law of Thermodynamics, the interaction of momentum and energy are represented by:

$$
\begin{gathered}
m_{s}^{\ddagger}=-m_{g}^{\ddagger}=p_{g} \frac{\partial \phi_{s}}{\partial x}-C_{d}\left(v_{s}-v_{g}\right) \\
e_{s}^{\ddagger}=-e_{g}^{\ddagger}=m_{s}^{\ddagger} v_{s}-h\left(T_{s}-T_{g}\right)-\left(p_{s}-\beta_{s}\right) \frac{\phi_{s} \phi_{g}}{\mu_{c}}\left(p_{s}-p_{g}-\beta_{s}\right) .
\end{gathered}
$$

The drag coefficient, $C_{d}$, and the interphase heat transfer coefficient, $h$, are exchange coefficients for momentum and energy phase interactions and depend on local flow conditions based on Reynolds number scaling. These constitutive laws are discussed in the next section. Model closure is obtained using a nonporous solid thermoelastic equation of state fit to shock Hugoniot data. Additionally, the ideal gas law is used for the gas phase equation of state.

\section{Constitutive Equations}

Critical constitutive relationships to the mixture model are the drag and heat transfer phase interactions and the compaction rate law for the low density foam. Polyester and polyurethane foam cylindrical plug sections, provided by B. Skews (University of Witwaterstrand), were tested in a transparent flow channel. Pressure drop measurements at low to high flow rates were taken in the determination of permeability of the foams and the Reynolds number scaling of the interphase drag. At high air flow rate, drag forces induce compaction of the porous material and visual measurement of uniaxial strain is sufficient for estimating the effective mechanical properties of the foam materials.

For nonshocked flow, pressure drop in rigid porous materials is related to flow velocity by the Forcheimer equation ${ }^{7}$ :

$$
\frac{\Delta p}{\delta}=\frac{\mu}{\kappa} V+\frac{c \rho_{8}}{\sqrt{\kappa}} V^{2}
$$

where $\delta$ is the length over which pressure drop occurs, $\mu$ is the gas viscosity, $\rho_{g}$ is the gas density, $\kappa$ is the foam permeability and $c$ is an inertial constant (typically a value $\sim$ 1.). The fundamental length scale associated with the gas flow is $\sim \sqrt{\mathrm{x}}$ and a local Reynolds number is defined as $R e=\rho_{g} V \sqrt{\mathrm{x}} / \mu$; thus the pressure drop is recast in terms of a pressure coefficient $C_{p}=\Delta p / \frac{1}{2} \rho_{g} V^{2}$ :

$$
C_{p}=\left(\frac{2 c \delta}{\sqrt{\kappa}}\right)+\left(\frac{2 \delta}{\sqrt{\kappa}}\right) / R e .
$$

Figure 1 displays a representative data fit of equation 8 for gas flow into polyester foam. The permeability of this material was measured as $\kappa=0.29 \times 10^{-4} \mathrm{~cm}^{2}$ and the inertial constant was determined to be $c=0.31$. Similar data was obtained for the polyurethane foam and the permeability was estimated to be $K=0.29 \times 10^{-5} \mathrm{~cm}^{2}$ and $\mathrm{c} \sim 10.4$. The interphase momentum was then determined as outlined in Reference 1. Having established appropriate Reynolds number scaling, the interphase heat transfer is represented by a fluidized bed correlation (see Reference 1).

The mechanical properties of the low density foams were estimated by measurement of the uniaxial strain on the plug subjected to high gas flow rate. As described by Gibson and Ashby ${ }^{8}$, the mechanical behavior of these foam materials consists of a linear elastic region (within a :ew percent of strain), an elastic buckling range to $80-90 \%$ of strain and the upper limit of material densification. In the flow experiments, strains were measured in the elastic buckling region; hence the effective stress of the porous material is approximated by a constant effective yield stress, $Y_{\text {eff. }}$. Using a force balance and mass conservation, this stress state is inferred from uniaxial compression data (induced by the drag forces of gas flow) by the following relationship:

$$
Y_{\text {eff }}=\Delta p / 2 \phi_{s}^{0} \frac{\Delta \delta}{\delta} .
$$


Figure 2 displays ( $^{n}$ paction data for a polyester foam material. The effective elastic buckling stress is estimated to be $\sim 1.07 \mathrm{MPa}$ and for polyurethane $Y_{\text {eff }}$ is estimated to be $\sim 0.8 \mathrm{MPa}$. It is noted that the effective bulk modulus is $K_{\text {eff }} \sim Y_{\text {eff }} / 3 \sim 0.25 \mathrm{MPa}$, which is consistent with shock Hugoniot measurement by Henderson ,et al. ${ }^{9}$

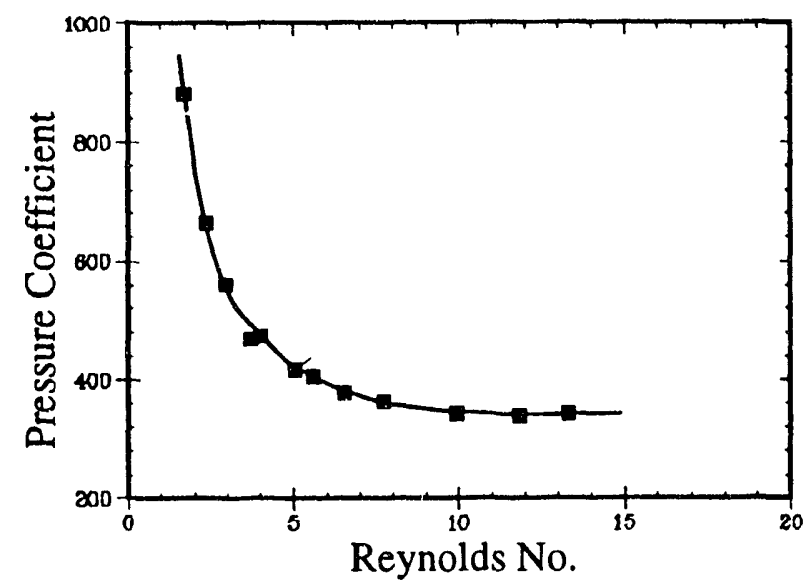

Figure 1. $C_{p}$ vs. Re for polyester foam.

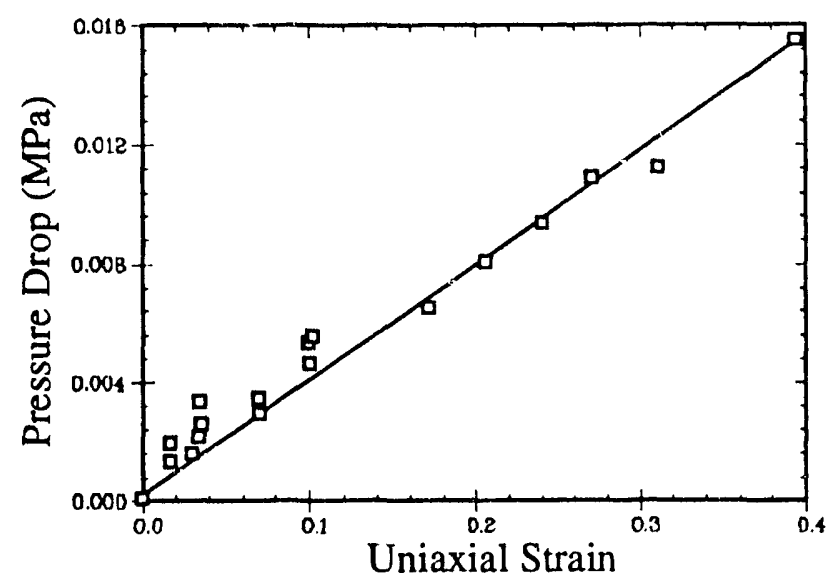

Figure 2. Compaction data for polyester foam.

\section{Numerical Solution}

Appropriate numerical methods to resolve these highly stiff multiphase equations have been previously reviewed by Baer, Benner, Gross and Nunziato ${ }^{10}$. This study uses an adaptive finite element numerical method. In this Eulerian approach, spatial derivatives are discretized using central differences and the resulting set of ordinary differential equations is solved using a stiff ODE solver. To stabilize the hydrodynamic calculation, a minimal amount of numerical viscosity is required to prevent numerical dispersion. An insert/delete adaptive noding method has been incorporated to enhance the numerical accuracy for resolving contact surfaces and shock fronts. This greatly reduces the effects of numerical diffusion. Up to eight levels of refinement (each level is a mesh reduction of a factor of two) is sufficient to capture the desired accuracy with approximately 200 adaptive elements.

\section{Numerical Calculations and Comparison to Experiment}

Using the aforementioned model, one-dimensional numerical simulations of shock tube experiments by B.W. Skews and colleagues ${ }^{3,4}$ and Onodera, et al. ${ }^{5}$ were conducted. The computational domain was taken to be $100 \mathrm{~cm}$ long and the initial high pressure air driver pressure was specified consistent with experimental incident shock Mach number, $M_{\mathbf{s}}$. Preliminary numerical calculations were done for $M_{s}=1.25$ interactions on wall-supported foams of varied length. Reference 10 reports preliminary calculations and a comparison to experiment. Revised numerical calculations. with greater element refinement, has demonstrated that all of the wave features are well-resolved and modeled as described by multiphase mixture theory.

Numerical calculations for a $\mathrm{M}_{\mathrm{s}}=1.4$ shock interacting with a low density $(0.038 \mathrm{~g} / \mathrm{cc})$ polyester foam of length $7 \mathrm{~cm}$ are shown in Figures 3 and 4, which display the temporal and spatial variations of pressure and solid volume fraction. After the shock hits the foam, a reflected wave passes back into the driver gas and a compaction wave occurs in the foam as gas permeates into the porous material imparting momentum to the solid phase. Compaction wave behavior is clearly seen in Figure 4. As the compaction wave interacts with the rigid boundary, stagnation of material produces a large pressure rise. Reflection of this pressure pulse produces a second reflected wave. This wave initially has a dispersive front but steepens to a reflected shock as supported by the rebounding foam. Its amplitude is greater than the first reflection off of the foam/air interface. Reflections within the foam layer produce a third pressure wave of low amplitude.

In the shock tube experiments by B. Skews et al. ${ }^{4}$, several pressure transducers were used to probe the wave fields. Figure 5 is a direct comparison of the multiphase theory to the experimental measurements at locations $T_{4}=4.3 \mathrm{~cm}$ and $T_{7}=10.3 \mathrm{~cm}$ from the wall, representing conditions interior and exterior to the foam layer. Clearly, the pressure histories are well described by the 
theoretical model. The calculated vs. measurement of pressure at the wall are also shown in Figure 5 . At this shock condition, the reflected wall pressure is amplified by a factor of $\sim 10$.

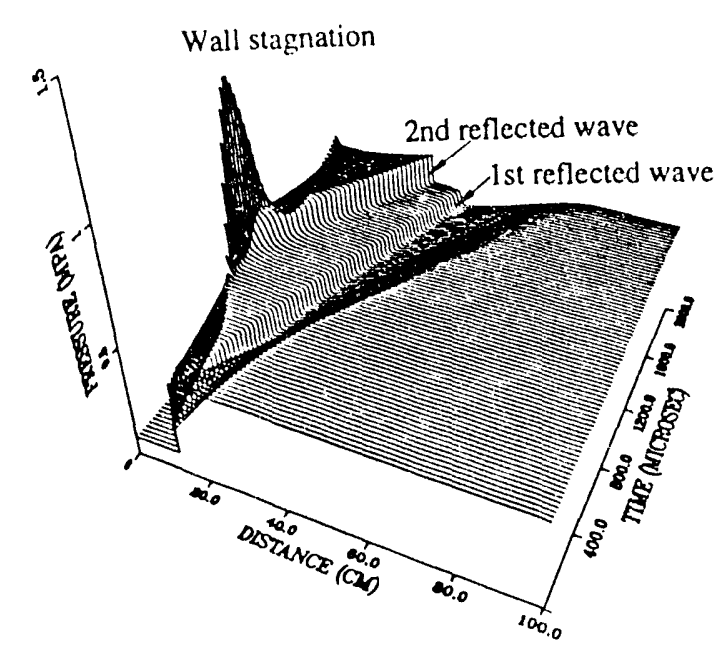

Figure 3. Pressure wave fields during shock interaction

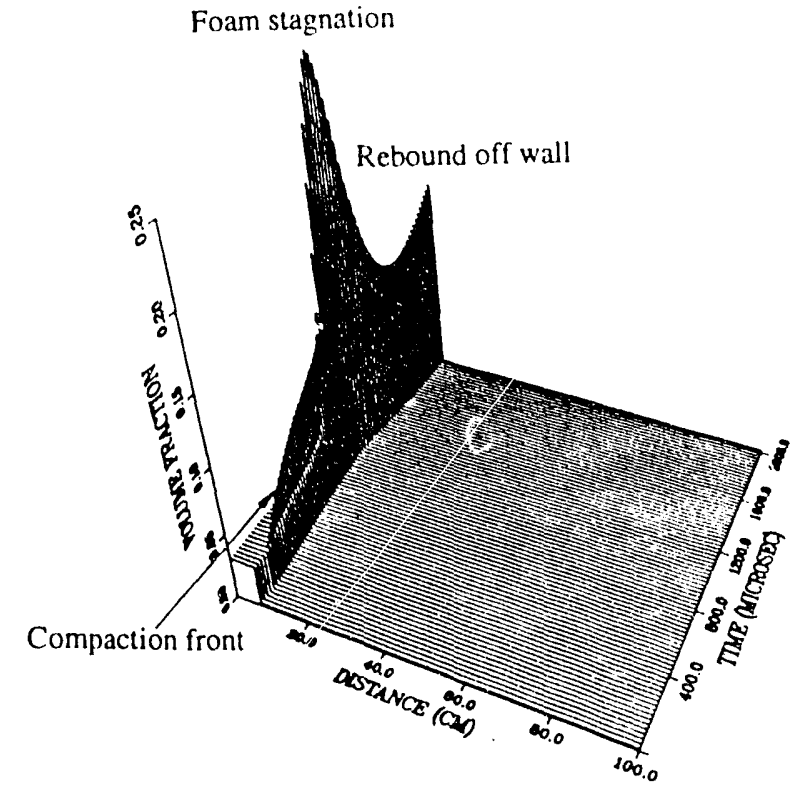

Figure 4. Solid volume fraction during shock interaction.
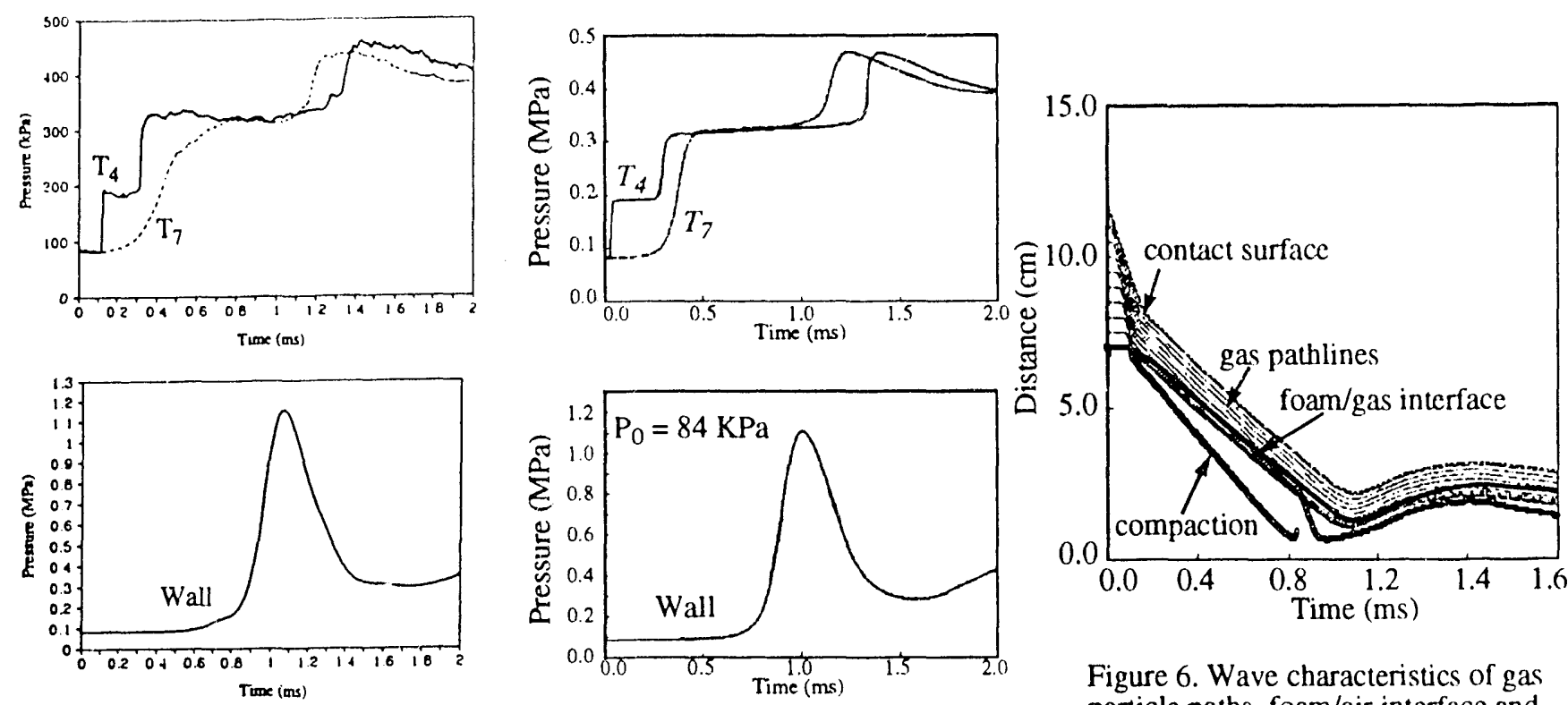

Figure 5. Comparison of experimental and calculated pressure histories.

Figure 6. Wave characteristics of gas particle paths, foam/air interface and compaction wave front.

To better characterize the wave fields, particle paths are shown in Figure 6 . The foam/air interface and the compaction wavehead are also displayed. Note that the gas pathlines penetrate into the foam layer at a depth of $\sim 2 \mathrm{~mm}$ from the foam/air interface. The compaction wave proceeds into the foam layer faster than the motion of the foam/air interface. At a later time, relaxation of the foam material is clearly evident.

Although experimental shock tube studies have not investigated the effect of initial gas pressure in the foam material, numerical simulations were done for $M_{s}=1.4$. Figure 7 displays the pressure histories at $\mathrm{T}_{4}, \mathrm{~T}_{7}$ and at the wall at ambient pressures of $84 \mathrm{KPa}, 50 \mathrm{KPa}$, and $400 \mathrm{KPa}$. (The ambient pressure for the shock tube experiment displayed in Figures 5 was $84 \mathrm{KPa}$ ). At lower pressure the wave fields are distinctly dispersive and the wall pressure is of a reduced amplification. At $400 \mathrm{KPa}$ the reflected waves quickly steepen. Clearly, the gas pressure in the foam layer has a significant effect which is indicative of multiphase wave behavior. 

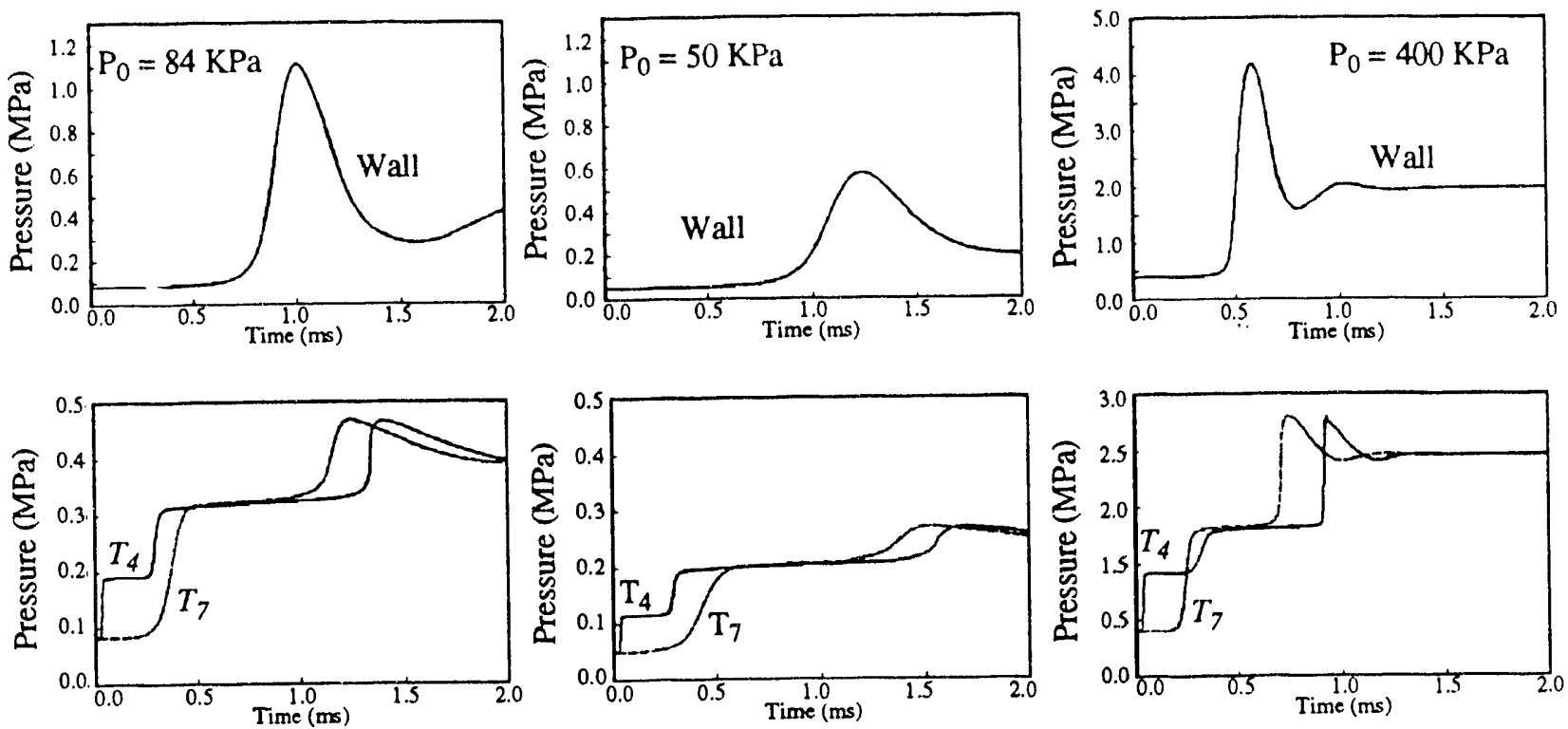

Figure 7. Pressure histories for $\mathrm{M}_{s}=1.4$ shock interaction on foams at varied ambient pressure.

In extended shock tube experiments, multiple foam layers (two layers $7.6 \mathrm{~cm}$ each) were impacted at an $\mathrm{M}_{\mathrm{s}}=1.51$. Figure 8 shows a comparison of the multiphase model to the experimental work of Skews and Seitz ${ }^{11}$. The transducer locations are in the foam layer at distances $6.3 \mathrm{~cm}, 3.3 \mathrm{~cm}$ and $0 \mathrm{~cm}$ from the wall. The inflection in the numerical prediction of the wall pressure (at the peak pressure) is due to the separation of layers upon reflection off the wall.
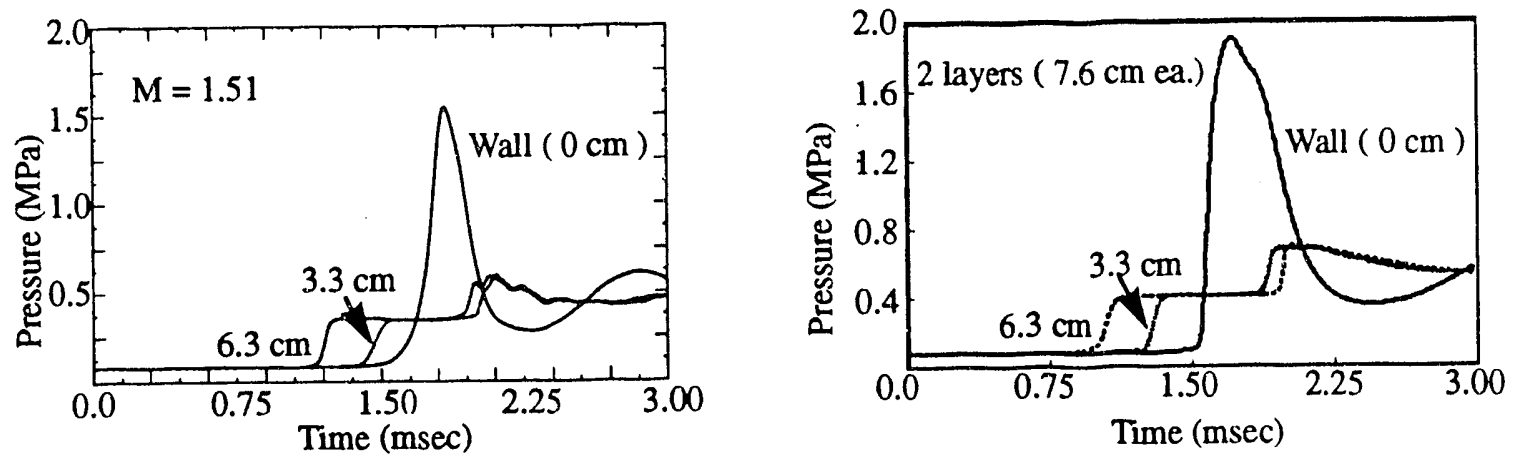

Figure 8. Experimental (left) vs. numerical (right) pressure histories of shock interaction on multiple foam layers.

As a final study, numerical calculations are presented for $\mathrm{M}_{\mathrm{s}}=1.28$ normal shock interaction on an unsupported polyurethane foam layer of thickness $2.5 \mathrm{~cm}$. An overlay of the pressure wave field in distance-time space is shown in Figure 9. The foam layer is approximately $40 \mathrm{~cm}$ from the location of the driver section. After the normal shock impinges on the unsupported foam, a reflected wave is produced and a compaction wave traverses the foam layer. A lower amplitude compression wave emerges from the trailing edge of the foam layer and acceleration of the foam causes steepening of the transmitted wave. Figure 10 displays a direct comparison of the pressure histories described by multiphase theory at locations consistent with the pressure measurements by Onodera, et al ${ }^{5}$. Pressure gauge 1 is located $29 \mathrm{~cm}$ in front of the foam layer and gauges 2,3 and 4 are upstream of the foam layer at distances $1.3 \mathrm{~cm}, 13.8 \mathrm{~cm}$ and $26.3 \mathrm{~cm}$, respectively. Consistent with the experimental observations, a two step wave pressure wave field evolves near the trailing edge of the foam layer (gauge 2), and the transmitted pressure wave monitored by gauges 3 and 4 has a reduced amplitude. 


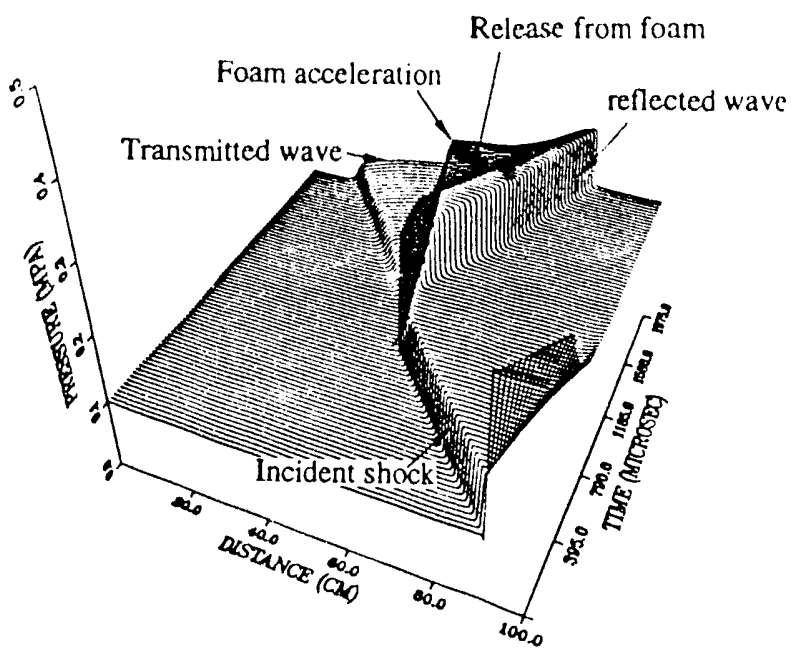

Figure 9. Pressure wave field associated with shock interaction on a unsupported foam layer.
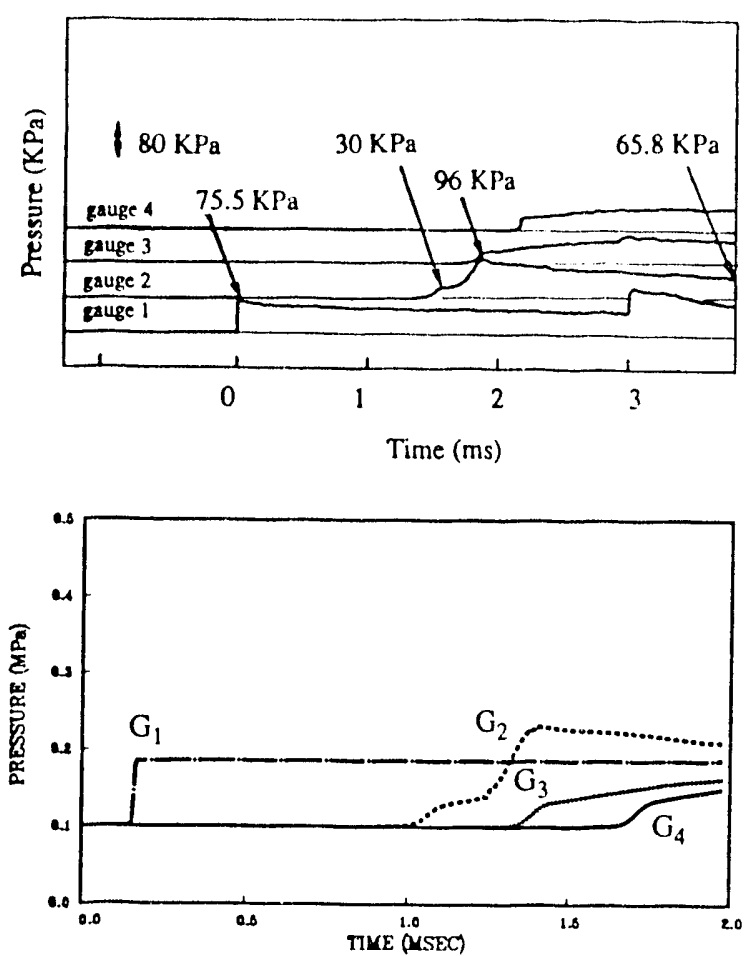

Figure 10. Comparison of experimental (top) and calculated (hottom) pressure histories at specified locations

\section{Summary}

In this study, a multiphase mixture theory has been successfully applied to describe several shock tube experiments. A variety of shock states and experimental configurations have been replicated and continuum mixture theory has been shown to describe well the shock-induced flow in low density foam. Numerical modeling can provide details of the complex wave behavior in highly distended materials.

\section{Acknowledgment}

I would like to thank Prof B. Skews and coworkers (University of Witwatersrand, S.Africa) for sharing their shoxk tube experimental data and providing the foam materials for the flow measurcments. I also thank F. Chavez (SNL/NM) for assembling and conducting the flow experiments and A. Kraynik (SNI/NM) for his assistance with the foam mechanics. This work was performed at Sandia National Laboratories supported by the US Dent. of Energy under contract \#DE-AC04-76DP00709.

\section{References}

1 Baer, M. R. and Nunziato, J. W., "A Two-Phase Mixture Theory for the Deflagration-to-Detonation Transition (DDT) in Reactive Granular Materials," International Journal of Multiphase Flow, Vol 12, no. 6, 1986, pp. 861-889.

2 Baer, M.R. and Nunziato, J.W., "Compressive Combustion of Granular Materials Induced by Low-Velocity Impact," Ninth Symposium (International) on Detonation, OCNR 113291-7, August 1989, pp. 293-305.

3 Skews, B. W. , "The Reflected Pressure Field in the Interaction of Weak Shock Waves With a Compressible Foam," Shock Waves, Vol. 1, no. 3., 1991, pp. 205-211.

4 Skews, B. W.,Atkins, M. D. and Seitz, M. W., "Gas Dynamic and Physical Behavior of Compressible Porous Foams Struck by Weak Shock Waves," Shock Waves, Ed. K. Takayama, Springer-Verlag, Heidelberg, Germany, 1992.

5 Onodera, H., Ben-Dor, G and Takayama, K., "Normal Shock Wave Interaction With and Penetration Through an Unsupported Porous Wall," to appear in Shock Waves, 1993.

6 Baer, M. R., "Numerical Studies of Dynamic Compaction of Inert and Energetic Granular Materials," Journal of Applied Mechanics, Vol. 55, 1988, pp. 36-43.

7 Collins, R.E.,Flow of Fluids Through Porous Materials, Penn Well Books and Pub. Company, Tulsa OK, 1976.

8 Gibson, L. J. and Ashby, M., Cellular Solids-Structure and Properties, Pergamon Press, 1988.

9 Henderson, L. F., Virgona, R. J., Di, J. and Gvozdeva, L. G., "Refraction of a Normal Shock Wave from Nitrogen into Polyurethane Foam," in Current Topics in Shock Waves, Ed. Y. W. Kim, American Institute of Physics, New York, NY, pp. 814-818, 1990.

10 Baer, M. R., Benner, R. E., Gross, R. J., and Nunziato, J. W., "Modeling and Computation of Deflagration-t()Detonation Transition (DDT) in Reactive Granular Materials," Lectures in Applied Mathematics, Americin Mathematical Society, Vol. 24, 1986, pp. 479-498.

11 Baer, M. R., "A Numerical Study of Shock Wave Reflection on Low Density Foam," Shock Waves, Vol. 2, pp 121124.

12 Skew's, B. and Seitz, M. personal communication. (1992). 

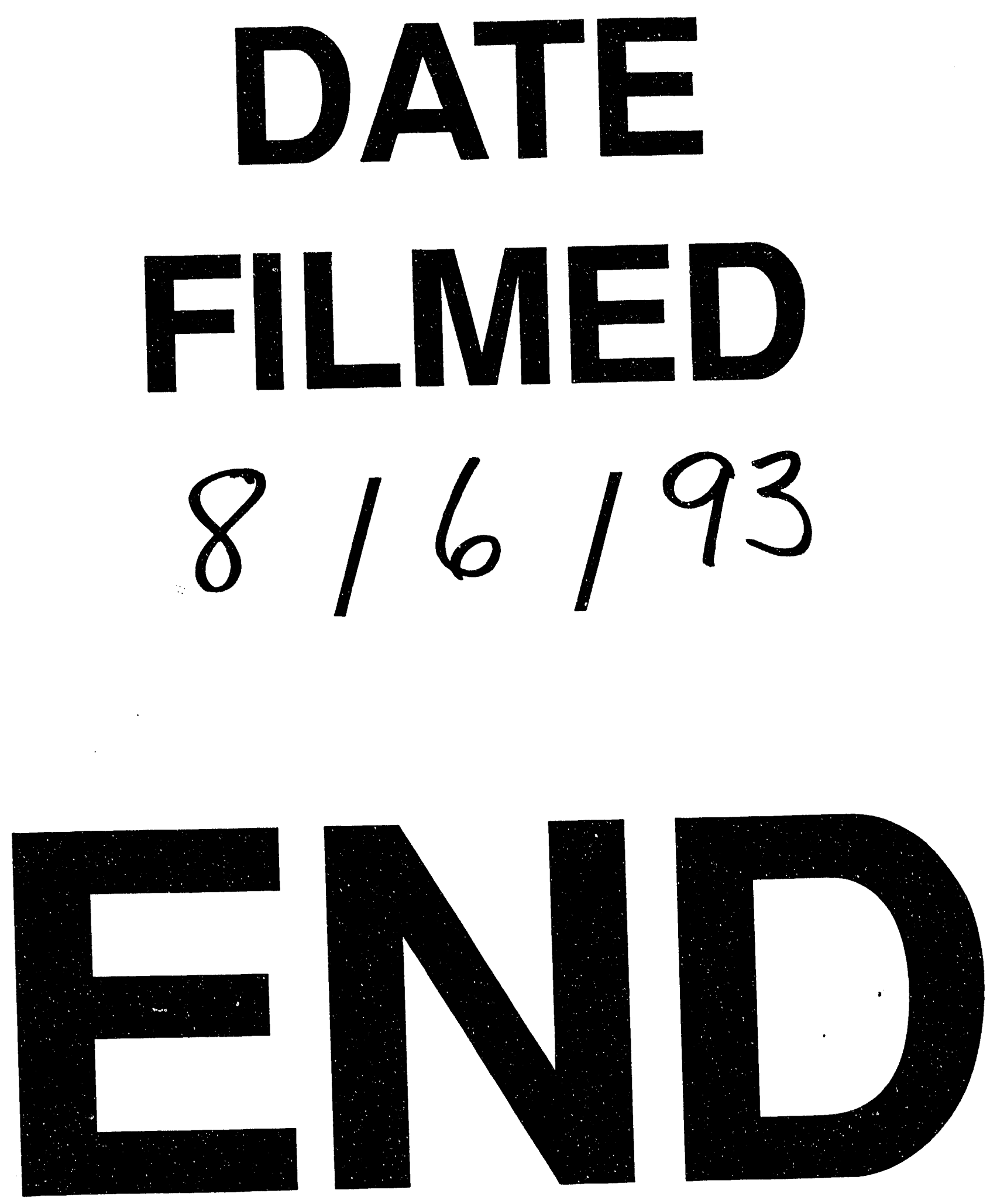
